\title{
Explaining the decrease in ISM lithium at super-solar metallicities in the solar vicinity
}

\author{
G. Guiglion ${ }^{1}$, C. Chiappini ${ }^{1}$, D. Romano ${ }^{2}$, F. Matteucci ${ }^{3,4,5}$, F. Anders ${ }^{1}$, M. Steinmetz ${ }^{1}$, I. Minchev ${ }^{1}$, \\ P. de Laverny ${ }^{6}$, and A. Recio-Blanco ${ }^{6}$ \\ ${ }^{1}$ Leibniz-Institut für Astrophysik Potsdam (AIP), An der Sternwarte 16, 14482 Potsdam, Germany \\ e-mail: gguiglion@aip.de \\ 2 INAF, Astrophysics and Space Science Observatory, Via Gobetti 93/3, 40129 Bologna, Italy \\ 3 Dipartimento di Fisica, Sezione di Astronomia, Università di Trieste, Via G.B. Tiepolo 11, 34131 Trieste, Italy \\ ${ }^{4}$ INAF Osservatorio Astronomico di Trieste, Via G.B. Tiepolo 11, 34131 Trieste, Italy \\ 5 INFN Sezione di Trieste, Via Valerio 2, 34134 Trieste, Italy \\ ${ }^{6}$ Université Côte d'Azur, Observatoire de la Côte d'Azur, CNRS, Laboratoire Lagrange, France \\ Received 7 September 2018 / Accepted 7 February 2019
}

\begin{abstract}
We propose here that the lithium decrease at super-solar metallicities observed in high-resolution spectroscopic surveys can be explained by the interplay of mixed populations that originate in the inner regions of the Milky Way disk. The lower lithium content of these stars is a consequence of inside-out disk formation plus radial migration. In this framework, local stars with super-solar metallicities would have migrated to the solar vicinity and depleted their original lithium during their travel time. To obtain this result, we took advantage of the AMBRE catalog of lithium abundances combined with chemical evolution models that take into account the contribution to the lithium enrichment by different nucleosynthetic sources. A large proportion of migrated stars can explain the observed lower lithium abundance at super-solar metallicities. We stress that no stellar model is currently able to predict Li-depletion for these super-solar metallicity stars, and solar Li-depletion has to be assumed. In addition, no solid quantitative estimate of the proportion of migrated stars in the solar neighborhood and their travel time is currently available. Our results illustrate how important it is to properly include radial migration when chemical evolution models are compared to observations, and that in this case, the lithium decrease at larger metallicities does not necessarily imply that stellar yields have to be modified, contrary to previous claims in the literature.
\end{abstract}

Key words. Galaxy: abundances - Galaxy: stellar content - stars: abundances

\section{Introduction}

The chemical evolution of the Milky Way (MW) lithium abundance is still a matter of debate. Recent high-resolution spectroscopic surveys have reported that the interstellar medium (ISM) lithium content of the MW disk in the solar vicinity decreases at super-solar metallicities $([\mathrm{M} / \mathrm{H}]>0.0 \mathrm{dex})$. Delgado Mena et al. (2015) were the first to state that lithium tend to decrease for $[\mathrm{M} / \mathrm{H}]>0$, for which result they used high- resolution spectra. This result has been confirmed by Guiglion et al. (2016) using HARPS, FEROS, and UVES spectroscopic data from the AMBRE project (de Laverny et al. 2012). These data cover a wider range in metallicity, up tol $[\mathrm{M} / \mathrm{H}] \sim 0.45 \mathrm{dex}$. The lithium decrease at super-solar metallicity was then observed by Fu et al. (2018) using Gaia-ESO spectrocsopic survey data and by Bensby \& Lind (2018) based on high-resolution data from various spectrographs (such as MIKE and FEROS).

This decrease is puzzling because standard chemical evolution models point toward a monotonous increase in lithium with metallicity (Romano et al. 2001; Prantzos 2012). Fu et al. (2018) suggested a possible connection with a reduced nova outburst rate at super-solar metallicity. With the ad hoc assumption of a significant reduction of the $\mathrm{Li}$ yields above solar metallicity, Prantzos et al. (2017) reproduced such a decreaes in lithium abundance at super-solar metallicity. However, previous stellar nucleosynthesis studies pointed out an increase in lithium yields for $[\mathrm{M} / \mathrm{H}]>0$ (see, e.g., Karakas \& Lugaro 2016).

When we study the Galactic lithium evolution with metallicity, we have to keep in mind that lithium is easily depleted in stellar interiors throughout the life of the star. Moreover, some lithium can be produced at specific stages of stellar evolution. The stellar lithium content cannot be assumed to be representative of the Li abundance of the ISM material from which the star was formed. Because of the broad spread in lithium for a given metallicity, it is important to only consider dwarf stars with the highest lithium, corresponding to those that have destroyed at least their photospheric lithium and have synthesized none. In practice, when predictions from a Galactic chemical evolution model are compared to the observations, the upper envelope of the data for dwarf stars needs to be considered (this is believed to be closer to the original lithium in the ISM) as a function of metallicity.

In this paper, we provide an explanation for the lithium decrease observed in super-solar metallicity thin-disk stars based on the AMBRE lithium catalog of Guiglion et al. (2016), the Galactic chemical evolution model for the thin disk of Chiappini (2009), and our current understanding of the chemodynamic evolution of our Galaxy (Minchev et al. 2013, 2014a; Kubryk et al. 2015). In Sect. 2 we present lithium patterns in 
different MW disk populations, and in Sect. 3 we explain the decrease in lithium boundary at high metallicities as due to the presence of old stars $(\tau>5 \mathrm{Gyr})$ that originate in the very inner regions that have a lower lithium content than locally born solarvicinity stars.

\section{ISM lithium abundance in the MW disk}

We used the AMBRE catalog of lithium abundances from Guiglion et al. (2016). This catalog contains chemical abundances of lithium and their non-local thermal equilibrium (NLTE) corrections, derived homogeneously from ESO HARPS, FEROS, and UVES spectra. The atmospheric parameters have been derived by Worley et al. $(2012,2016)$ and De Pascale et al. (2014), and can be found in the publicly available catalog from Guiglion et al. (2018), with detailed chemical abundances of $s$ - and $r$-process elements. In this study, we selected dwarf stars $(\log (g)>3.5)$ for which lithium abundance and NLTE corrections were available, excluding upper limits. We focused on the best stellar parameters and lithium abundances, that is, on stars with AMBRE QUALITY_FLAG=0 (see Worley et al. (2012) for more details on this parameter). We rejected the coolest dwarf stars $\left(T_{\text {eff }}<5300 \mathrm{~K}\right)$ with a deeper convective zone (which results in higher lithium destruction). The median signal-to-noise ratio $(\mathrm{S} / \mathrm{N})$ of our final sample is 105 .

As recently demonstrated by Anders et al. (2018), the use of the dimensionality reduction algorithm t-SNE ${ }^{1}$ method (see Sect. 3) allows a more robust definition of subpopulations in abundance space. For this technique to work, it is crucial to confine the analysis to narrow regions in atmospheric-parameter space to avoid spurious abundance trends induced by differences in atmospheric parameters. Although we do not have all the detailed chemical information to be able to compute the t-SNe subpopulations for our sample, we can use those found in Anders et al. (2018) for guidance because the two samples (the sample studied by Anders et al. and ours) are consistent in terms of abundance range and atmospheric parameters (see their Fig. 2, using data from Delgado Mena et al. (2017)).

Therefore, we chemically identified the MW disk populations in the $[\alpha / \mathrm{Fe}]$ vs. $[\mathrm{M} / \mathrm{H}]$ chemical space (e.g., Fuhrmann 1998, 2011; Minchev et al. 2015; Martig et al. 2016; Kawata \& Chiappini 2016 using the same definitions as in Guiglion et al. (2016, see their Fig. 10 and their Sect. 6.2 for more details). We find that the thin and chemical thick disks are fully consistent with those of Anders et al. (2018). The main difference is that through the t-SNE method, Anders et al. (2018) also identified a metal-rich $\alpha$-rich population (so-called mr $\alpha$ r, Adibekyan et al. 2011), which we here now also include by defining as super-solar metallicity stars all thin disk stars with $[\mathrm{M} / \mathrm{H}]>$ 0.2 dex and solar $\alpha$-element abundance. Once again, this is possible because the atmospheric parameters and chemical abundances in AMBRE are consistent with the study of Delgado Mena et al. (2017) (see Guiglion et al. 2018 for more details). The resulting chemical patterns are presented in the left panel of Fig. 1.

After we defined the sub-populations and in order to trace the lithium abundance of the ISM as a function of metallicity, we followed the same procedure as Lambert \& Reddy (2004) and Guiglion et al. (2016): we binned the AMBRE data in metallicity, and computed an average lithium abundance for the six

\footnotetext{
1 t-distributedStochastic Neighbour Embedding (Van der Maaten \&
} Hinton 2008). stars with the highest lithium abundance in a given metallicity bin. The NLTE lithium abundance as a function of $[\mathrm{M} / \mathrm{H}]$ of our sample is shown in the right panel of Fig. 1, with different colors corresponding to the four populations identified in the left panel. The resulting trends in ISM lithium abundance for the four populations are overplotted with the colored lines, with error bars corresponding to the standard deviation of the six stars in each metallicity bin.

The thick-disk lithium envelope first rises from $A_{\mathrm{Li}}^{\mathrm{NLTE}} \sim$ 2 dex at $[\mathrm{M} / \mathrm{H}]=-1 \mathrm{dex}$ to $A_{\mathrm{Li}}^{\mathrm{NLTE}} \sim 2.4 \mathrm{dex}$ at $[\mathrm{M} / \mathrm{H}]=$ -0.2 dex. The thin-disk lithium envelope shows an increase of about 1 dex and reaches the meteoric abundance at solar metallicity. The envelope for the mror stars reaches that of the thin disk at solar metallicity and clearly decreases by about 0.20 dex at $[\mathrm{M} / \mathrm{H}] \sim+0.3 \mathrm{dex}$. In the metal-rich solar- $\alpha$ population, the lithium content is clearly lower than in the mror stars and in the thin-disk stars with solar metallicity. The trends presented here remain the same when the slope of the chemical separation is slightly varied and the bin size of the metallicity axis is changed. In addition, low statistics is unlikely to degrade the observed trends because the proportion of stars $(4 \%)$ with $A_{\mathrm{Li}}^{\mathrm{NLTE}}>3.0$ dex is the same for both $-0.1<[\mathrm{M} / \mathrm{H}]<+0.1 \mathrm{dex}$ and $[\mathrm{M} / \mathrm{H}]>+0.1$ dex. The lithium decrease is therefore not due to a poor sampling of the metal-rich populations.

\section{Explaining the lithium decrease at super-solar metallicity}

The answer to why in the metal-rich $[\alpha / \mathrm{Fe}]-$ rich (green) and super-metal-rich solar- $\alpha$ (magenta) populations $\mathrm{Li}$ decreases with metallicity involves three different processes that are simultaneously at work: (1) the Li-enrichment in the ISM by different stellar sources (e.g., Prantzos et al. 2017), (2) the Li depletion throughout the evolution of the star (e.g., Lagarde et al. 2012), and (3) the fact that the more metal-rich stars are not the youngest stars, as has been shown observationally (e.g., Casagrande et al. 2011; Trevisan et al. 2011; Anders et al. 2017a) and explained theoretically by the process of radial migration (e.g., Roškar et al. 2008; Schönrich \& Binney 2009; Minchev et al. 2013; Bird et al. 2013; Vera-Ciro et al. 2014; Martinez-Medina et al. 2017).

In Fig. 2 we illustrate the points made above and investigate the feasibility of our explanation quantitatively as well. Although the Li-enrichment is mostly well understood, large uncertainties still prevent a detailed quantification of the effects of Li-depletion and radial migration from pure theoretical arguments. In the case of the two latter processes, we therefore use empirical data to guide our estimates.

\subsection{Lithium enrichment}

In Fig. 2 we show chemical evolution tracks for three different radial bins centered on $R=2,4$, and $8 \mathrm{kpc}$ and the data described in Fig. 1. The theoretical curves are color-coded by age, corresponding to the color bar. These tracks were computed using the Galactic chemical evolution model of Chiappini (2009) (see also Minchev et al. 2013 for more details). In this model, the Galactic disk is divided into rings of $2 \mathrm{kpc}$ width that evolve independently (i.e., without radial gas flows) in an inside-out fashion (Matteucci \& Francois 1989). This allows us to track the contributors to the ISM lithium enrichment with time and radius. The nucleosynthesis prescriptions for lithium adopted in the Chiappini (2009) model are the same as in the best model of 

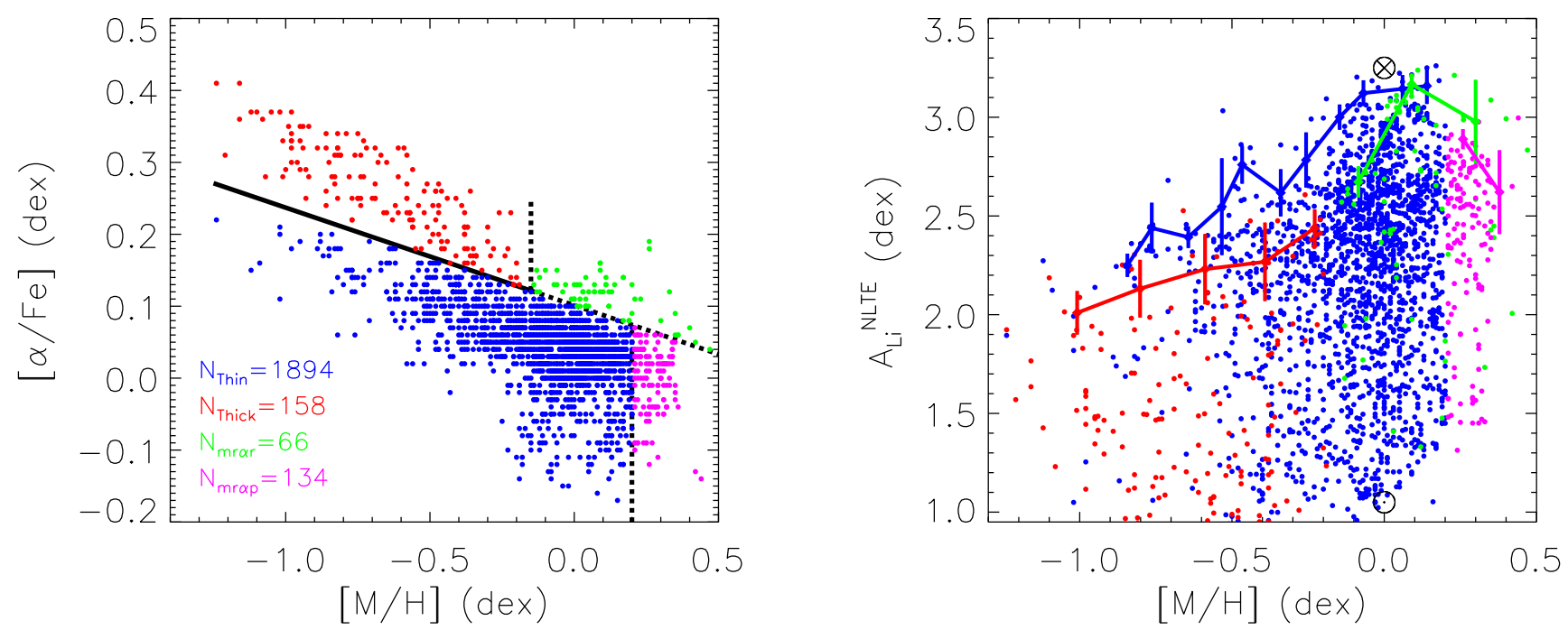

Fig. 1. Left panel: $[\alpha / \mathrm{Fe}]$ vs. $[\mathrm{M} / \mathrm{H}]$ patterns for the thin-disk (blue), canonical thick-disk (red), metal-rich $[\alpha / \mathrm{Fe}]-$ rich stars (green), and supermetal-rich solar- $\alpha$ stars (magenta). The black lines show how we identified these four MW populations. Right panel: AMBRE NLTE lithium abundance vs. metallicity for the corresponding four populations using the same color-code as in the left panel. For a given population, we plot the maximum lithium abundance using the same method as Guiglion et al. (2016). The meteoric lithium abundance, which is not affected by from stellar evolution, is also shown $(\otimes)$.

Romano et al. (1999) ${ }^{2}$. We are not interested in a detailed discussion of the Li nucleosynthesis here (see Prantzos et al. 2017; Romano et al. 1999, 2001, where a discussion can be found), but in showing the general behavior of a chemical evolution model that assumes an inside-out formation (i.e., higher star formation efficiencies (SFE) toward the inner regions of the Galaxy), and takes into account the several sources of Li nucleosynthesis.

The chemical evolution tracks clearly show that the onset of the strong Li increase, mainly powered by the contribution of long-lived sources such as AGBs and novae (depending on the details of the chemical evolution model adopted) occurs at higher metallicities for the innermost regions ${ }^{3}$ (a consequence of the higher star formation rates; see, e.g., Matteucci \& Brocato 1990; Chiappini et al. 2001; Hou et al. 2000; Minchev et al. 2013; this is illustrated by the horizontal arrow in Fig. 2).

\subsection{Lithium depletion throughout the stellar evolution}

In addition to lithium evolution in the ISM, lithium is depleted in stellar interiors during the main-sequence phase. No stellar model is currently able to reproduce the correct lithium depletion in the Sun or predict Li-depletion in $[\mathrm{Fe} / \mathrm{H}]>0$ stars. In stellar models that include rotation and thermohaline mixing, the lithium depletion does not appear to increase for more metal-rich

\footnotetext{
2 In the model of Romano et al. (1999), several lithium sources in addition to Big Bang nucleosynthesis were considered: cosmic rays impacting on ISM nuclei, Type II supernovae (through the $v$-process), carbon stars, massive asymptotic giant branch stars (AGBs), and novae. Lithium astration in stars of all masses was also taken into account. It was found that a long-lived stellar source was needed to reproduce the observations, with novae being recently confirmed as a critical ingredient of the model (see Izzo et al. 2015).

3 We here take the $2 \mathrm{kpc}$ curve as our innermost example, but more extreme models assuming higher star formation efficiencies could be constructed in which the Li knee is displaced to even higher metallicities. However, in this case, the final metallicity would be beyond $[\mathrm{Fe} / \mathrm{H}]=0.6$, which is the value already reached for the $R=2 \mathrm{kpc}$ curve, and therefore unrealistic given that no star has so far been observed at such a high metallicity.
}

stars (see the models of Lagarde et al. 2012). In Fig. 2 we therefore assumed a typical solar depletion for the more metal-rich stars in populations green and magenta as well. The arrow in Fig. 2 at $[\mathrm{Fe} / \mathrm{H}]=0.5$ is then not arbitrary, but indicates the difference between the pre-solar cloud value measured in meteorities and the solar photospheric lithium value (both values are also illustrated in Fig. 2), which implies a 2.2 dex depletion during $4.5 \mathrm{Gyr}$ of evolution (illustrated by the dashed arrow at $[\mathrm{Fe} / \mathrm{H}]=0)$.

\subsection{Effect of radial migration}

As clearly shown by both observations (e.g., Casagrande et al. 2011; Trevisan et al. 2011; Anders et al. 2017a) and chemodynamical models (Minchev et al. (2013, 2014b), the most metal-rich stars (those with $[\mathrm{Fe} / \mathrm{H}]>0.25$ ) are typically 5-10 Gyr old. Therefore all of them will have depleted their lithium by large amounts (as discussed in $b$ ). It follows that the lithium decrease at super-solar metallicities that is observed in the solar neighborhood might be explained by radial migration, that is, that these stars were not born locally (Minchev et al. 2013, 2014b, 2018; Anders et al. 2018, Chiappini et al., in prep.). These stars will have systematically lower lithium than young stars that were born at the solar radius (here illustrated by the $R=8 \mathrm{kpc}$ curve).

\section{Conclusion}

We have chemically characterized four Milky Way disk populations using a classical chemical criterion in the well-known $[\alpha / \mathrm{Fe}]$ vs. $[\mathrm{M} / \mathrm{H}]$ plane: a thin-disk, a chemical thick-disk, a metal-rich $\alpha$-rich population, and a super-metal-rich solar- $\alpha$ population $([\mathrm{M} / \mathrm{H}]>0.2 \mathrm{dex})$. These populations and chemical patterns are consistent with the recent analysis of Anders et al. (2018) and Chiappini et al. (in prep.). For these four distinct populations, we took advantage of the AMBRE catalog of lithium abundances from Guiglion et al. (2016) to trace the lithium abundance in the ISM as a function of metallicty. We showed that stars migrating from the innermost parts of the Galactic disk 


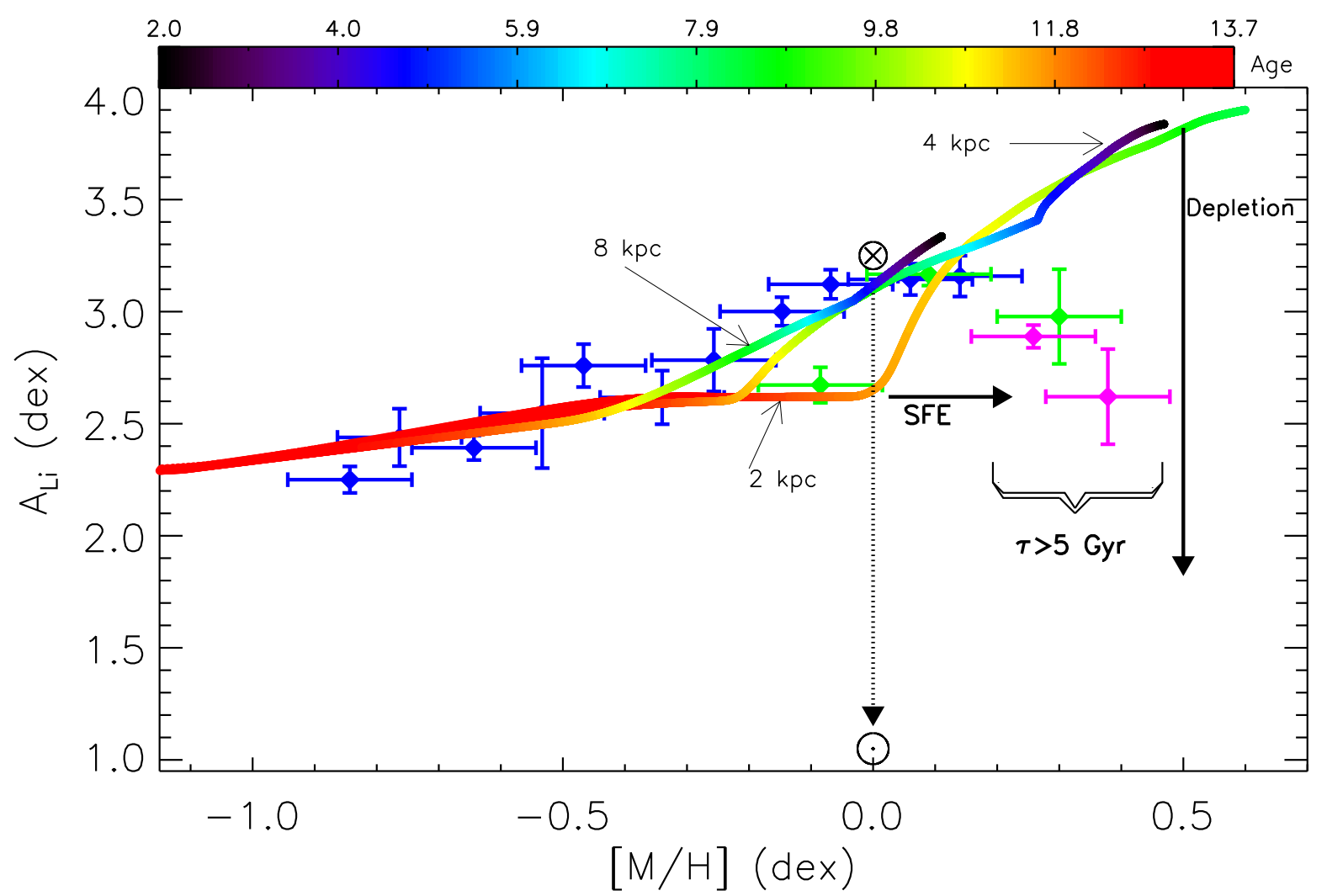

Fig. 2. Lithium abundance vs. metallicity for the thin-disk (blue dots), a metal-rich $\alpha$-rich population (green dots) and a super-metal-rich solar- $\alpha$ population (pink dots) characterized in Fig. 1. We show a Galactic chemical evolution model of the thin disk (Chiappini 2009), for three different galactocentric bins of $2 \mathrm{kpc}$ width (centered at 2, 4, and $8 \mathrm{kpc}$ ), color-coded by age. First, inside-out chemical evolution models predict the knee of the lithium tracks to occur at higher metallicities for the innermost zones because star formation is more efficient (illustrated by the horizontal thick arrow). Second, the internal depletion of lithiun in the Sun is illustrated by the vertical dashed arrow, from the meteoric abundance to the photospheric one. Third, current data suggest super-metal rich stars to be older than $\sim 5 \mathrm{Gyr}$, which implies a further lithium decrease due to depletion. These old ages can be understood in the context of radial migration. However, because there is no lithium depletion model at super-solar metallicities, we have assumed this depletion to be equal to the solar one (vertical thick arrow).

(birth radii $<2 \mathrm{kpc}$ ) will have low lithium. If confirmed by stellar age-dating, this finding would rule out the need of modifying stellar yields at super-solar metallicity.

This work illustrates the effect that more robust definitions of MW stellar populations (allowed by methods such as the tSNE; Anders et al. 2018; Chiappini et al., in prep) can have on the recent claim that a $\mathrm{Li}$ decline in super-solar metallicity stars found by many authors (Delgado Mena et al. 2015; Guiglion et al. 2016; Fu et al. 2018) would imply a revision of the lithium stellar yields at high metallicities. As shown in Anders et al. (2018) and Chiappini et al. (in prep.), the most metalrich thin-disk stars have most probably migrated from inner disk regions (as has been indicated by previous observations such as Casagrande et al. 2011; Trevisan et al. 2011, and Anders et al. 2017b; see the discussion in Chiappini 2015), and cannot be modeled as part of a local thin disk without taking into account the fact that stars move out of their birth places, as is clearly shown by chemodynamical models (Minchev et al. 2013, 2014b, 2018). It is also consistent with the kinematics found in supersolar metallicity stars in the solar neighborhood, see, e.g., Wojno et al. (2018).

Acknowledgements. The authors acknowledge the anonymous referee for the comments and suggestions that improved the readability of the paper. GG and CC thank Corinne Charbonnel for useful discussions. CC acknowledge the DFG project number 283705981: "Analysing the chemical fingerprints left by the first stars: chemical abundances in the oldest stars". ARB acknowledges finan- cial support form the ANR 14-CE33-014-01. IM acknowledges support by the Deutsche Forschungsgemeinschaft under the grant MI 2009/1-1.

\section{References}

Adibekyan, V. Z., Santos, N. C., Sousa, S. G., \& Israelian, G. 2011, A\&A, 535, L11

Anders, F., Chiappini, C., Minchev, I., et al. 2017a, A\&A, 600, A70

Anders, F., Chiappini, C., Rodrigues, T. S., et al. 2017b, A\&A, 597, A30

Anders, F., Chiappini, C., Santiago, B. X., et al. 2018, A\&A, 619, A125

Bensby, T., \& Lind, K. 2018, A\&A, 615, A151

Bird, J. C., Kazantzidis, S., Weinberg, D. H., et al. 2013, ApJ, 773, 43

Casagrande, L., Schönrich, R., Asplund, M., et al. 2011, A\&A, 530, A138 Chiappini, C. 2009, in The Galaxy Disk in Cosmological Context, eds.

J. Andersen, B. Nordströara, \& J. Bland-Hawthorn, IAU Symp., 254, 191

Chiappini, C. 2015, EAS Pub. Ser., 73, 309

Chiappini, C., Matteucci, F., \& Romano, D. 2001, ApJ, 554, 1044

de Laverny, P., Recio-Blanco, A., Worley, C. C., \& Plez, B. 2012, A\&A, 544, A126

De Pascale, M., Worley, C. C., de Laverny, P., et al. 2014, A\&A, 570, A68

Delgado Mena, E., Bertrán de Lis, S., Adibekyan, V. Z., et al. 2015, A\&A, 576, A69

Delgado Mena, E., Tsantaki, M., Adibekyan, V. Z., et al. 2017, A\&A, 606, A94

Fu, X., Romano, D., Bragaglia, A., et al. 2018, A\&A, 610, A38

Fuhrmann, K. 1998, A\&A, 338, 161

Fuhrmann, K. 2011, MNRAS, 414, 2893

Guiglion, G., de Laverny, P., Recio-Blanco, A., et al. 2016, A\&A, 595, A18

Guiglion, G., de Laverny, P., Recio-Blanco, A., \& Prantzos, N. 2018, A\&A, 619, A143

Hou, J. L., Prantzos, N., \& Boissier, S. 2000, A\&A, 362, 921 
G. Guiglion et al.: Explaining the decrease in ISM lithium at super-solar metallicities

Izzo, L., Della Valle, M., Mason, E., et al. 2015, ApJ, 808, L14

Karakas, A. I., \& Lugaro, M. 2016, ApJ, 825, 26

Kawata, D., \& Chiappini, C. 2016, Astron. Nachr., 337, 976

Kubryk, M., Prantzos, N., \& Athanassoula, E. 2015, A\&A, 580, A126

Lagarde, N., Decressin, T., Charbonnel, C., et al. 2012, A\&A, 543, A108

Lambert, D. L., \& Reddy, B. E. 2004, MNRAS, 349, 757

Martig, M., Minchev, I., Ness, M., Fouesneau, M., \& Rix, H.-W. 2016, ApJ, 831, 139

Martinez-Medina, L. A., Pichardo, B., Peimbert, A., \& Carigi, L. 2017, MNRAS 468,3615

Matteucci, F., \& Brocato, E. 1990, ApJ, 365, 539

Matteucci, F., \& Francois, P. 1989, MNRAS, 239, 885

Minchev, I., Chiappini, C., \& Martig, M. 2013, A\&A, 558, A9

Minchev, I., Chiappini, C., \& Martig, M. 2014a, A\&A, 572, A92

Minchev, I., Chiappini, C., Martig, M., et al. 2014b, ApJ, 781, L20

Minchev, I., Martig, M., Streich, D., et al. 2015, ApJ, 804, L9
Minchev, I., Anders, F., Recio-Blanco, A., et al. 2018, MNRAS, 481, 1645 Prantzos, N. 2012, A\&A, 542, A67

Prantzos, N., de Laverny, P., Guiglion, G., Recio-Blanco, A., \& Worley, C. C. 2017, A\&A, 606, A132

Romano, D., Matteucci, F., Molaro, P., \& Bonifacio, P. 1999, A\&A, 352, 117

Romano, D., Matteucci, F., Ventura, P., \& D’Antona, F. 2001, A\&A, 374, 646 Roškar, R., Debattista, V. P., Quinn, T. R., Stinson, G. S., \& Wadsley, J. 2008, ApJ, 684, L79

Schönrich, R., \& Binney, J. 2009, MNRAS, 396, 203

Trevisan, M., Barbuy, B., Eriksson, K., et al. 2011, A\&A, 535, A42

Van der Maaten, L., \& Hinton, G. 2008, J. Mach. Learn. Res., 9, 85

Vera-Ciro, C., D’Onghia, E., Navarro, J., \& Abadi, M. 2014, ApJ, 794, 173

Wojno, J., Kordopatis, G., Steinmetz, M., et al. 2018, MNRAS, 477, 5612

Worley, C. C., de Laverny, P., Recio-Blanco, A., Hill, V., \& Bijaoui, A. 2016, A\&A, 591, A81

Worley, C. C., de Laverny, P., Recio-Blanco, A., et al. 2012, A\&A, 542, A48 